\title{
Composition Table Of Moroccan Culinary Recipes.
}

\author{
Nisrine El Mir ${ }^{1}$, Naima Safsaf ${ }^{1}$, Aicha Alabridi ${ }^{1}$, Meryem Lazrak $^{1}$, Hanane Bouijij ${ }^{2}$, Laila El Ammari ${ }^{3}$, Naima \\ Saied $^{1}$, Hasnae Benkirane ${ }^{1}$, Khalid El Kari ${ }^{1}$ and Hassan Aguenaou ${ }^{1}$ \\ ${ }^{1}$ Ibn Tofaïl University-CNESTEN, Joint Research Unit in Nutrition, Health and Environment, Laboratory Biology and \\ Health', \\ ${ }^{2}$ Office National Des Oeuvres Universitaires Sociales et Culturelles'. \\ 'Ministry of Health, Moroccco'
}

\begin{abstract}
Good nutrition plays an essential role in our health. It decreases the risk of developing certain chronic diseases and thus increases life expectancy. This study is the first of its kind in Morocco, its objective is to determine the nutrient composition of the most consumed Moroccan culinary recipes. Thus, thanks to the calculation of the nutriscore, we can know which culinary recipe is the "healthiest".

Some of the most consumed Moroccan traditional dishes were collected in which carbohydrates, fats, and proteins were analyzed using the procedures of the Association of Official Analytical Chemists, then followed by a calculation of the food exchange lists per serving using the Wheeler method.

Variations in macronutrients, micronutrients (minerals and trace elements) and trans-fatty acid content, polyunsaturated and monounsaturated were found among the Moroccan dishes. The highest protein $(17.37 \mathrm{~g} / 100 \mathrm{~g})$ in Chicken, French Fries, and Cumin Tajine while the lowest $(0.06 \mathrm{~g} / 100 \mathrm{~g})$ in Orange and Carrot Salad. The highest carbohydrates $(42.68 \mathrm{~g} / 100 \mathrm{~g})$ in Tuna Pizza while lowest $(2.37 \mathrm{~g} / 100 \mathrm{~g})$ in tomato with pepper. And the highest lipids $(19.2 \mathrm{~g} / 100 \mathrm{~g})$ in meat with onion Tajine and the lowest $(0.6 \mathrm{~g} / 100 \mathrm{~g})$ in Harira. Meanwhile, the energy ranged between 35.7 and $352.4 \mathrm{Kcal} / 100$ $\mathrm{g}$ in the dishes. For each dish and according to the size of each serving, the exchanges of carbohydrates (starch), fats, and proteins (lean meat, medium fat, and high-fat meat) were calculated.

This study offers an opportunity - for health professionals, dietitians as well as consumers - to orchestrate with knowledge traditional dishes and ensuring leading dietary and medical nutritional therapy practices and patient self-control.
\end{abstract}

\section{Introduction}

Morocco is one of the first countries in North Africa to implement a national nutrition strategy that brings together four complementary strategic axes, namely food security, strengthening the nutrition component of the health program, training human resources, and establishing mechanisms for monitoring and evaluating this strategy.

To align with the objectives of this strategy, the Ministry of Health led during the year 2019[1], a National Nutrition Survey, which was guided by the Population Management/National Nutrition Program with the collaboration of the Planning and

Morocco and other developing countries are experiencing a very noticeable change in their eating habits [3], and some national studies in this direction confirm the shift from a cereal-based diet, of fruits and vegetables to a diet rich in saturated fats, cholesterol, sugar, and sodium.

Various published studies endorse the link between nutritional transition and the onset of * Corresponding author: nissrine.elmir@uit.ac.ma
Financial Resources Department and the Epidemiology and Disease Control Department, the National institute of hygiene and the designated Regional Nutrition Centre. It was funded by $\mathrm{WHO}$ (world healt organization, and UNICEF (United Nations Children Fund). [2]

The report of the survey revealed more recent information on the nutritional situation of the population of Morocco precisely in micronutrients more particularly iodine, iron, vitamin A, vitamin D, and folic acid. [2]

chronic diseases including obesity and micronutrient deficiencies

The present study aims to:

- Comparison of the nutritional intakes of the most consumed Moroccan dishes with the nutritional status of the Moroccan population. 
- Assess the Visibility and legibility on the Moroccan diet.

\section{MATERIALS AND METHODS:}

\section{Data collection:}

A total of 200 traditional dishes were chosen as the most consumed by Moroccans, they were prepared and cooked in the laboratory of the mixed nutrition unit according to the guidelines and protocol followed by "Food Table Composition Data" [4]
A H24 recall was carried out on 200 families in the Rabat Kenitra region to determine the 15 most widely eaten dishes including $(n=7)$ Main dishes, $(n=4)$ Side dishes $(n=5)$ salads.

\section{Composition of dishes}

The ingredients of our dishes were prepared by an average recipe determined by cookbooks most used by Moroccans and grandmother recipes collected during the $\mathrm{H} 24$ recall survey.

Table 1: Presents the list of ingredients designated for a sample preparation.

\begin{tabular}{l} 
Traditional dish \\
\hline Moroccan Salad \\
Orange and Carrot Salad \\
Harira (Moroccan soup) \\
Lentil Dish \\
Dried Bean Dish \\
Oven Grilled fish \\
Chicken Tagine with Potatoes \\
and Olives
\end{tabular}

Fish Tagine with potatoes and peppers

Zaalouk

Meat Tagine with prunes

Taktouka with peppers

Bean Soup

Couscous with Tefaya

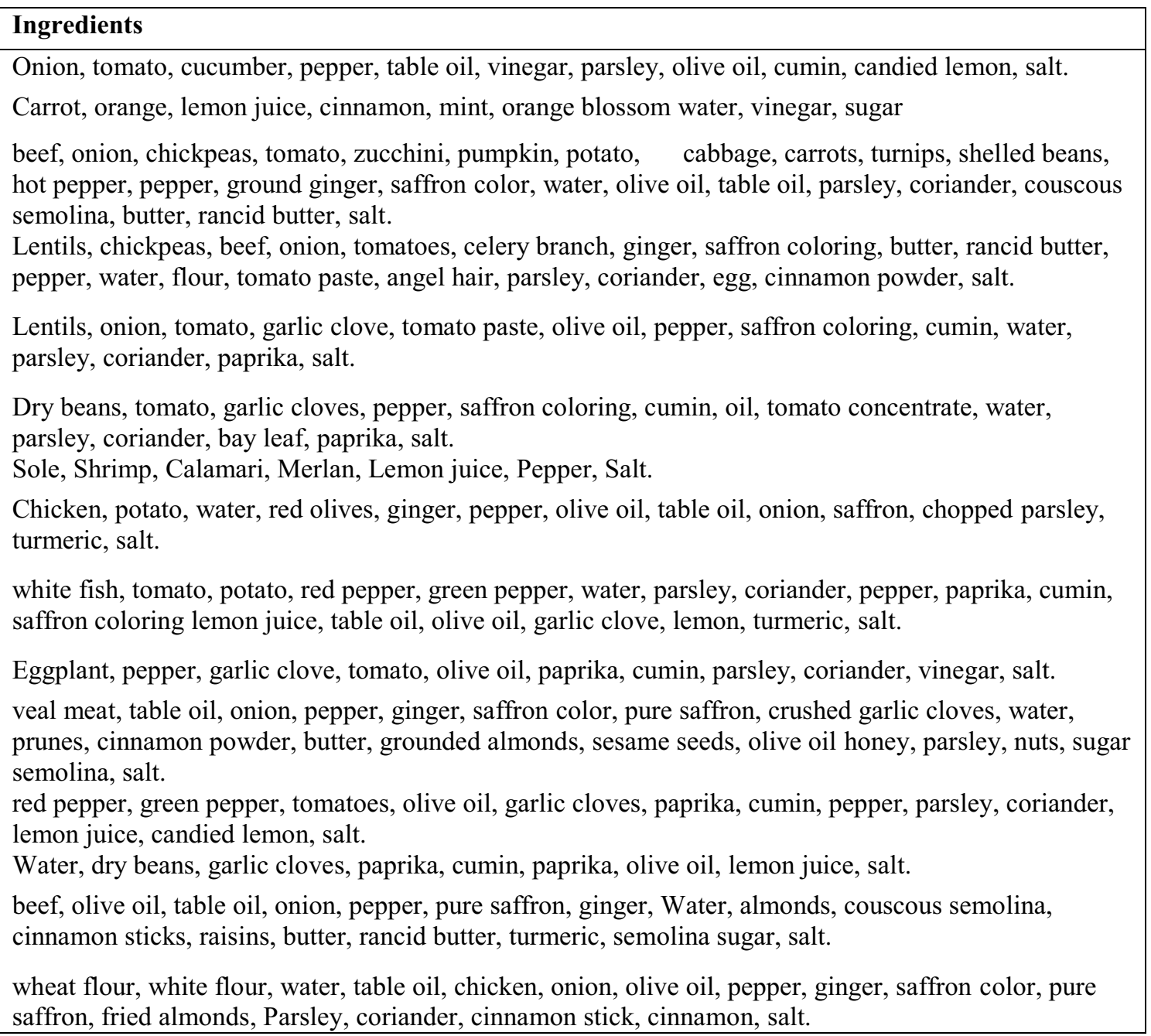

continously until a homogeneous dough was obtained using a mixing robot (Moulinex press FP824H10) [4].
Was performed in the kitchen laboratory following the Protocol and the "food composition data" [4] repository while we recorded the total weight of each sample. Then, each preparation was mixed 
The resulting paste was immediately placed in vacuum food bags and frozen at a temperature of $20 \mathrm{C}$ until analysis [4]

\section{Sample Analysis}

The analysis of iodine content was carried out according to the Iodometry method and the rest of the micronutrients was carried out by ICP/MS in an

\section{Results:}

\section{The nutritional intake of Iron:}

The analysis of the iron content of the 15 most consumed dishes showed different values from one recipe to another. Ranging from $241.83 \mathrm{mg} / 100 \mathrm{~g}$ in Taktouka with couscous peppers 7 vegetables with $5.5 \mathrm{mg} / 100 \mathrm{~g}$ and Moroccan salad with 4.05 $\mathrm{mg} / 100 \mathrm{~g}$. (Figure 1) accredited laboratory: the LC2A laboratory in Mohamadia.

The levels of vitamins D, B9, Retinol, and B carotene were determined by calculation of the mixed method (calculation of the nutritional composition of the recipes based on the Moroccan food tables and Ciqual) due to the excessive cost of analyses.

Odometry showed a near-absent iodine content on all analyzed dishes with a lower value of $0.025 \mathrm{mg} / 100 \mathrm{~g}$.

\section{Vitamin D intake:}

Of the 15 dishes, two Harira dishes and chicken tagine with potatoes and olives contain vitamin D but in small quantities $(0.1 \mathrm{ug} / 100 \mathrm{~g})$. (Figure 3)

\section{Dietary intake of iodine:}

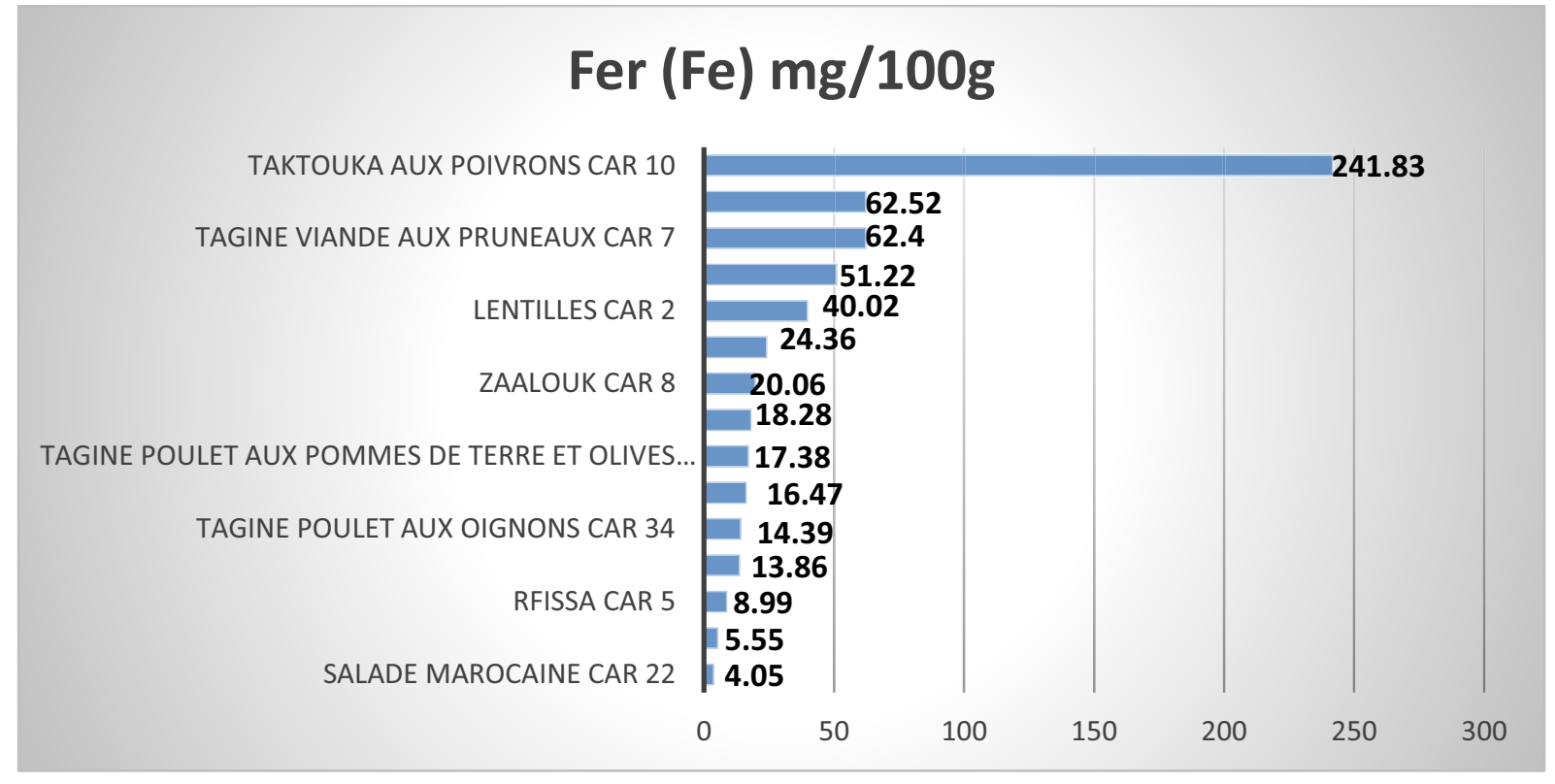

Figure 1: ug/100 content of iron in dishes from richest to lowest

\footnotetext{
* Corresponding author: nissrine.elmir@uit.ac.ma
} 


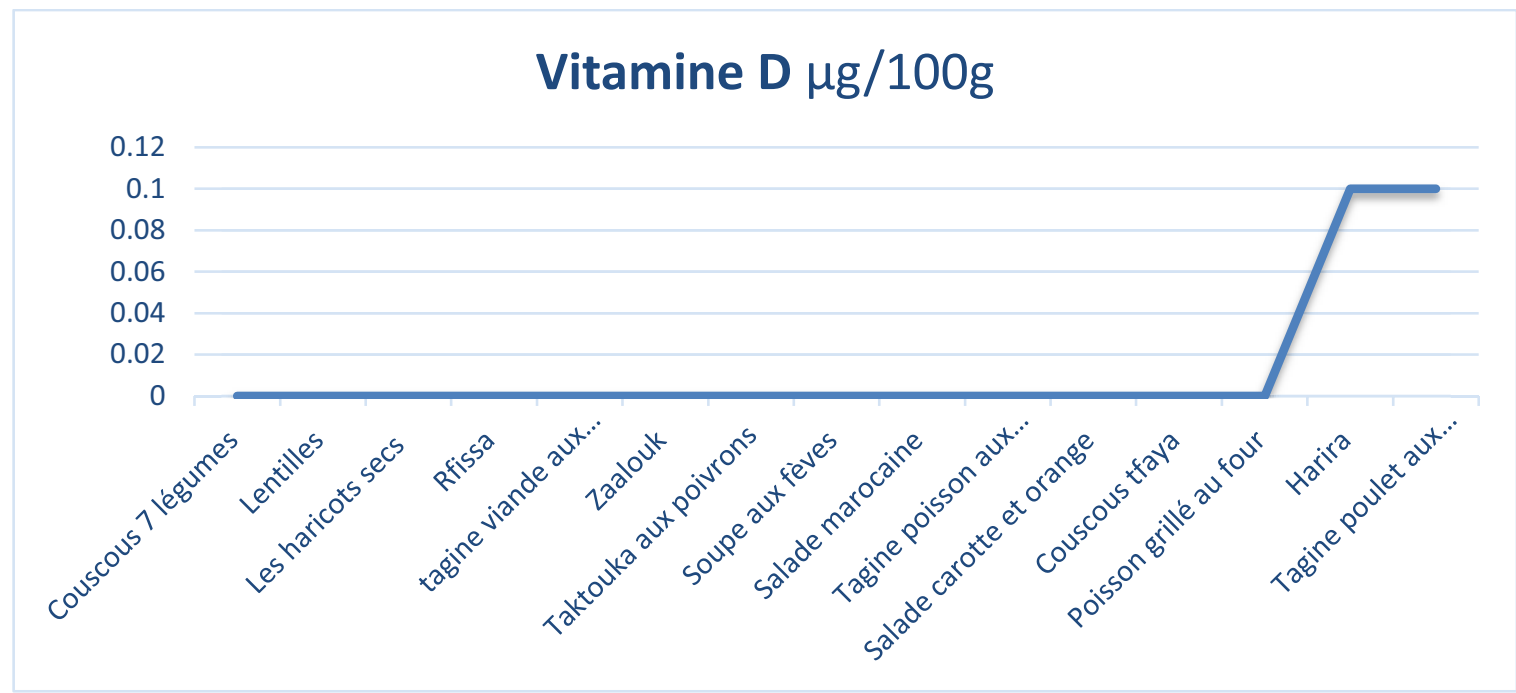

Figure 3: ug/100 vitamin D content of dishes from lowest to richest

\section{Folic acid intake:}

Vitamin B9 or folic acid is one of the watersoluble vitamins, it is also called «folates» this term recalling that $\mathrm{B} 9$ is abundant in green and leafy vegetables or explains the fact that it is found in different quantities at the level of the 15 dishes and even in fairly high quantities at the level of green beans with $48,5 \mathrm{ug} / 100 \mathrm{~g}, 88.3 \mathrm{ug} / 100 \mathrm{~g}$ in lentils and $98.9 \mathrm{ug} / 100 \mathrm{~g}$ found in bean soup. (Figure 4)

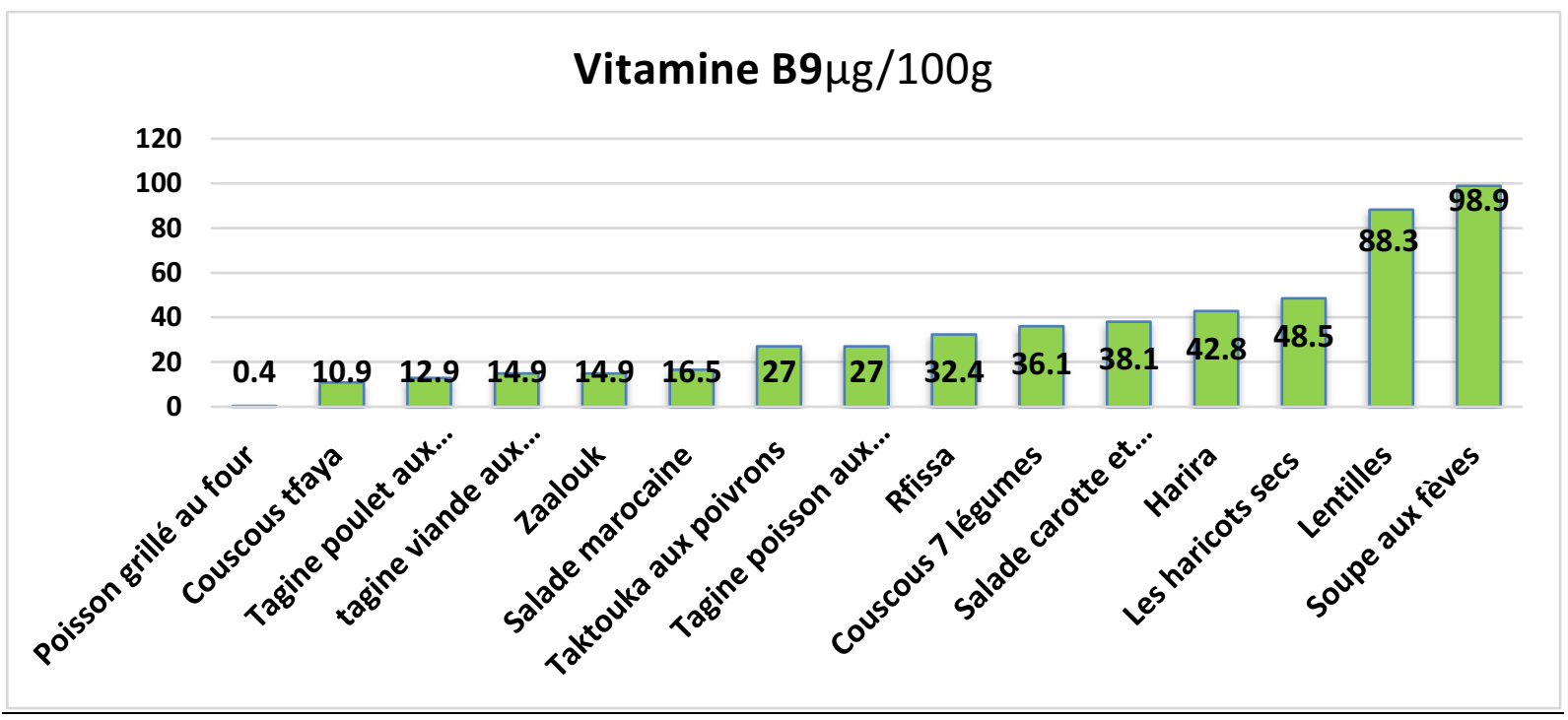

Figure 4: ug/100 content of vitamin B9 in dishes from lowest to richest

\section{Intake of Retinol and Beta carotene:}

Figures 5 and 6 show the levels of Retinol and Beta carotene at the level of our 15 dishes which are found respectively and with very high concentrations at the level of the Moroccan salad with a rate of $401,4 \mathrm{ug} / 100 \mathrm{~g}$ and with a concentration of $2530,2 \mathrm{ug} / 100 \mathrm{~g}$ beta carotene in the dish richest in iron taktouka tomato and pepper

*Corresponding author: nissrine.elmir@uit.ac.ma 


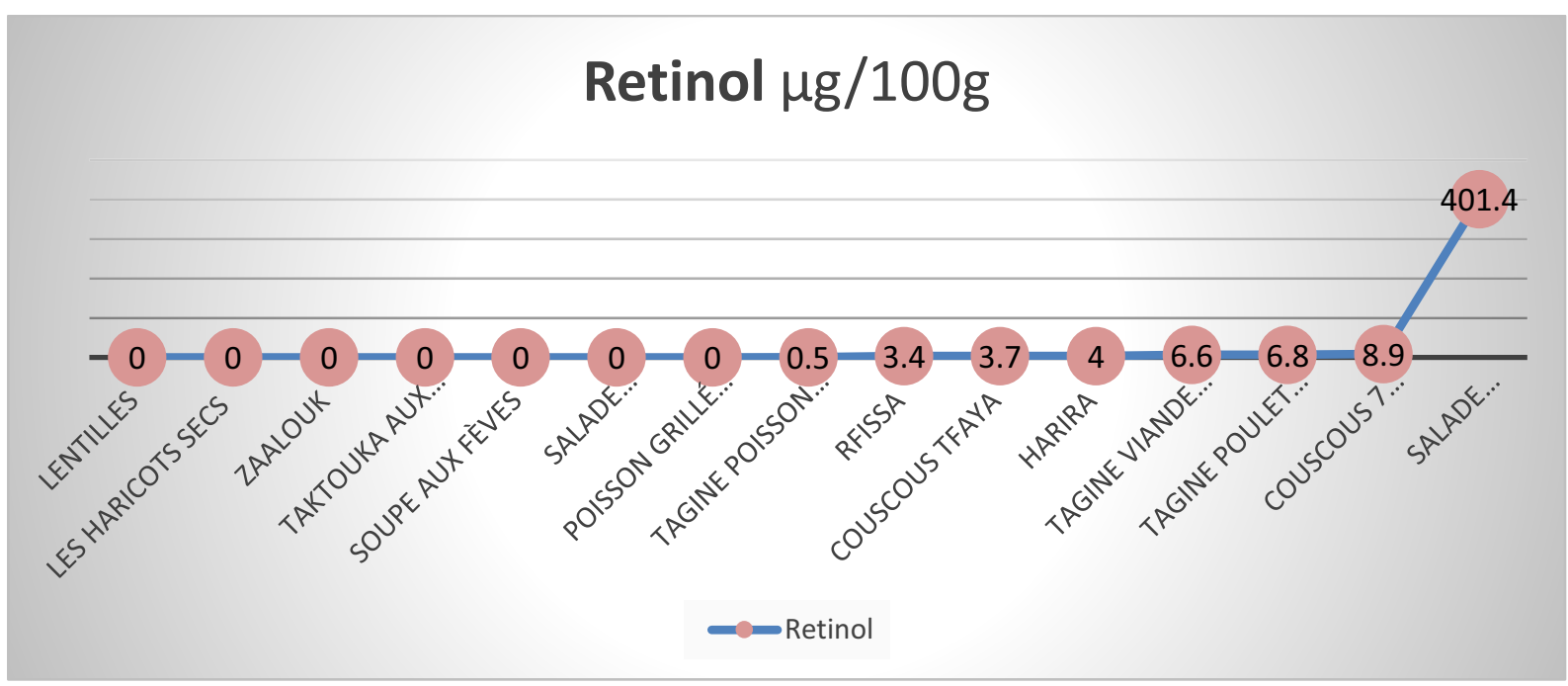

Figure 5: ug/100 content of Retinol from lowest to richest dish.

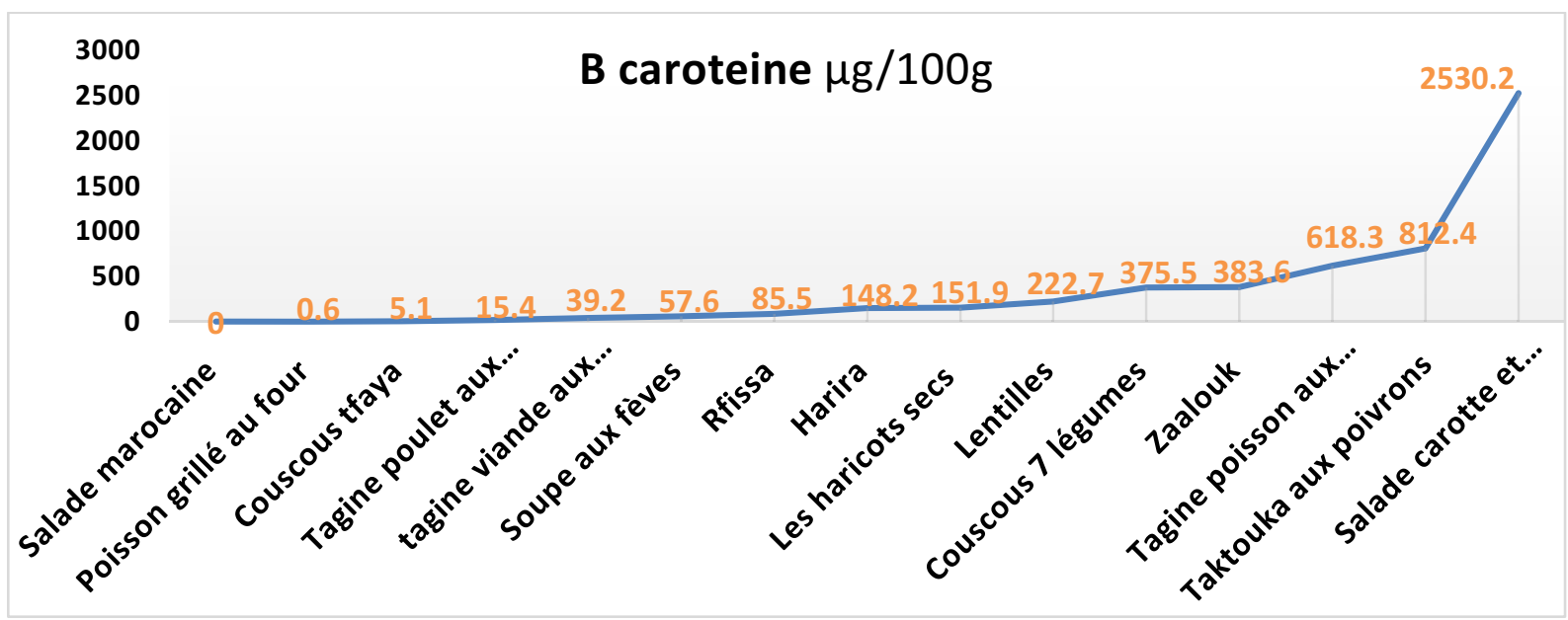

Figure 6: Beta carotene ug/100 content of dishes from lowest to richest

\section{Discussion}

The national survey showed that anemia and iron deficiency are still public health problems. [1]

The results of this study revealed that the mean iron content in the 15 dishes analyzed was $40.02 \mathrm{ug} / 100 \mathrm{~g}$, and with very high concentrations in most dishes, which are highly consumed and appreciated by most Moroccans and within the reach of all Moroccan socio-cultural classes, one notices, for example, the taktouka with a rate of $241,8 \mathrm{mg} / 100 \mathrm{~g}$ and also the lenses with $40,02 \mathrm{mg} / 100 \mathrm{~g}$ which can cover a large percentage of recommended daily intake of iron, while remaining acaloric, a similar consumption allows to have a balanced and acaloric diet for the Moroccan.

However, the survey found that $34.4 \%$ of women are anemic, of which $15.6 \%$ have moderate to severe anemia. Also, $30.4 \%$ of women have iron deficiency and $49.7 \%$ of anemic women have iron deficiency. Iron deficiency was also found in $11.9 \%$ of children and $23.7 \%$ of anemic children. The investigation showed, once again, that anemia and iron deficiency are two closely related markers $(\mathrm{p}<0.0001)$. [1]

This situation can be explained by the profound and rapid change that Morocco has experienced in recent decades[6], resulting from the growth of food industry production, fast food especially for people who work full time, and the presence of media that encourage the consumption of products that promote the appearance of several deficiencies and other metabolic disorders and thus the installation of a nutritional transition. [3]

The knowledge of vitamin D has greatly improved in recent years. Vitamin D can no longer 
be considered solely necessary for the prevention of rickets/osteomalacia. Its role in the prevention of peripheral osteoporotic fractures of the elderly is now well demonstrated (in association with calcium) and a great deal of epidemiological and experimental data are in favor of a role in the prevention of many diseases (certain cancers and autoimmune diseases, cardiovascular events and hypertension, sarcopenia of the elderly...)[7].

The assessment of vitamin D content in women of childbearing age showed that $30.7 \%$ were deficient in vitamin D and $47.9 \%$ were deficient in vitamin D [8] . The survey also showed that vitamin D deficiency affects only $3 \%$ of children aged 6 to 12 years while $27.3 \%$ have vitamin D deficiency. [8]

In our study the levels of vitamin D were very low and present only in two dishes, since the source of vitamin D is not always and sufficiently food, moderate exposure to the sun can provide a very high percentage of the intake recommended by the WHO.

Folic acid is a vitamin of group B whose needs increase during pregnancy $7 \%$ of women of childbearing age have a folic acid deficiency. However, the only data available in Morocco on folic acid status go back to the sentinel survey conducted in 2008, which reported a confirmed deficiency in about $23.9 \%$ of women of childbearing age. [9]

While our analysis of the most consumed dishes shows very high concentrations of folic acid and present in popular dishes and at the door of all socio-cultural classes, on the contrary lentils with $88,3 \mathrm{ug} / 100 \mathrm{~g}$ followed by bean soup with its very high rate of $98.9 \mathrm{ug} / 100 \mathrm{~g}$, seasonal consumption of bean soup may explain this deficiency, since generally, consumption of starchy and similar dishes increases greatly in winter.

Nutrition education and awareness in this direction could contribute to nutritional rebalancing. [3]

\section{Conclusion}

The results of this study show for the first time in Morocco that the most consumed compound meals contribute to a balanced Moroccan diet that covers daily nutritional intakes of essential micronutrients, without bringing so many calories, these are a caloric and recommended dishes.

\footnotetext{
*Corresponding author: nissrine.elmir@uit.ac.ma
}

The survey showed that vitamin A deficiency is $10.9 \%$, mostly moderate, and is more present in rural areas (12.9\%) compared to urban areas $(9 \%)$ among children while there are no data on vitamin A deficiency for women. [8]

In our study the calculation was established on the concentrations of retinol and beta carotenes that can improve the status of vitamin A, the content of Retinol was very remarkable in Taktouka with a concentration of $401,4 \mathrm{ug} / 100 \mathrm{~g}$, while the beta carotene content had fairly high concentrations on a skewer of 8 dishes usually served as a salad or side dish, per $148.2 \mathrm{ug} / 100 \mathrm{~g}$ in Hrira,383.6ug/100g in Zaalouk and $2530.2 \mathrm{ug} / 100 \mathrm{~g}$ in orange carrot salad.

That said, confirmation of street food that is ubiquitous in the city and yet convenient and especially cheap meets the needs not only of working people, but also of poor families[3], and which usually rests from a single dish unlike the Moroccan cuisine which is known by its meals in associated dishes: Salad +Side + main dish

The results of this study show for the first time in Morocco that the most commonly consumed compound dishes contribute to a balanced Moroccan diet that covers daily nutritional intakes of essential micronutrients, without bringing so many calories, these are a caloric and recommended dishes, while the national survey has stated that deficiencies are still present.

Morocco, therefore, and like many developing countries, is suffering the consequences of a deviation from the Mediterranean food model [3]. It is, therefore, necessary to accentuate efforts on different pillars namely: nutrition education, awareness, and enhancement of the Moroccan diet, to fight against these deficiencies that have a profound impact on the health and precisely of the mother than on that of the fetus during pregnancy. [3]

the Moroccan food profile is experiencing intense and alert changes resulting from the growth of urbanization, economic development, the increase in the production of the food industry[3], and the presence of media that encourage the consumption of products that promote the appearance of several 
deficiencies and other metabolic disorders and thus the establishment of a nutritional transition[3].

\section{REFERENCES}

\section{1}

[1] Ministry of health, national nutrition strategy, (2011-2019), Morocco

[2] Ministry of health, national program of nutrition, (2019), Morocco

[3] F.Allali,International Journal of Medicine and Surgery,4,68-71, Nutrition Transition in Morocco(2017)

[4] FAO, H. Greenfield,D.A.T. Southgate,Données sur la composition des aliments,308,(2007)

[5] WHO,Worksop, Calcul des valeurs nutritionnelles (VN) des recettes et des valeurs manquantes, Tunisie,(2018)

[6] Ministry of Agriculture and Maritime Fisheries, situation alimentaire et nutritionnelle au Maroc, Avril 2007 this work presents a starting point for the analysis of other traditional dishes consumed in Morocco.

[7]J.Bacchetta,B.Ranchin,L.Dubourg,P.Cochat,Archi ves de Pédiatrie,17,1687-1695,Vitamine D : un acteur majeur en santé ?,(2010)

[8] PNN, Enquête Nationale sur la Nutrition, Diversité alimentaire, Carence en Fer, Carence en Vitamine A, Carence en lode, (2019).

[9]N.Wolak,M.Zawrotniak,M.Gogol,A.Kozik,M.Rapa la-Kozik,Mini Reviews in Medicinal Chemistry,17,1075-1111,Vitamins B1, B2, B3 and B9-Occurrence, Biosynthesis Pathways and Functions in Human Nutrition,(2017)

\footnotetext{
* Corresponding author: nissrine.elmir@uit.ac.ma
} 